\title{
Benefits of adjuvant treatment including trastuzumab in HER2- positive pT1a-bNOMO breast cancer: a systematic review and meta-analysis
}

\author{
Hye Yoon Lee ${ }^{1}$, In-Soo Shin ${ }^{2}$, Chai Hong Rim $^{3}$ \\ ${ }^{1}$ Division of Breast and Endocrine Surgery, Department of Surgery, Ansan Hospital, Korea University, Gyeonggido, Republic of Korea; ${ }^{2}$ Department \\ of Education, College of Education, Dongguk University, Seoul, Republic of Korea; ${ }^{3}$ Department of Radiation Oncology, Ansan Hospital, Korea \\ University, Gyeonggido, Republic of Korea \\ Contributions: (I) Conception and design: CH Rim; (II) Administrative support: None; (III) Provision of study materials or patients: None; (IV) \\ Collection and assembly of data: HY Lee; (V) Data analysis and interpretation: IS Shin; (VI) Manuscript writing: All authors; (VII) Final approval of \\ manuscript: All authors. \\ Correspondence to: Chai Hong Rim, MD, PhD. Department of Radiation Oncology, Ansan Hospital, Korea University, 123 Jeokgeum-ro, Danwon-gu, \\ Ansan, Gyeonggi-do 15355, Republic of Korea. Email: crusion3@naver.com.
}

Background: Although trastuzumab has been shown to be beneficial for treating patients with human epidermal growth factor receptor 2 (HER2)-positive breast cancer, information regarding its benefits is limited to very low-risk cases with tumours $\leq 1 \mathrm{~cm}$ and without lymphatic metastases (pT1abN0). The present meta-analysis integrates information from literature and determines the benefit of trastuzumab in pT1abN0, HER2-positive breast cancer patients.

Methods: PubMed, MEDLINE, and EMBASE databases were searched for studies published before Sep 30, 2019. Our primary endpoint was tumor recurrence, whether provided as overall or distant recurrences.

Results: Seven studies involving 1,181 patients with pT1abN0, HER2-positive breast cancer were included in the systemic review. The median follow-up periods ranged from 37 to 78 months. The patients in the trastuzumab arm had generally inferior profiles such as higher rate of T1b, grade 3, and hormone negative cases, among available studies. Concomitant chemotherapy was more commonly applied in the trastuzumab arm (75-100\% vs. 0-42\%), and the hormone therapy application was similar in both arms (20-66\%). In a pooled analysis of seven available studies, patients treated with trastuzumab had less overall recurrence relative to controls, with an odds ratio of 0.201 [95\% confidence interval (CI): $0.100-0.404, \mathrm{P}<0.001$ ]. Five studies were available for a pooled analysis of distant recurrence. Although the results were not significant $(\mathrm{P}=0.115)$, distant recurrence did not occur in 237 patients treated with trastuzumab, but did occur in 16 out of 436 control patients. The odds ratio for distant recurrence was 0.328 (95\% CI: 0.082-1.311).

Conclusions: The adjuvant treatment including trastuzumab was shown to reduce overall recurrence. Distant recurrence may also be reduced, as it did not occur among the 237 patients who underwent trastuzumab treatment.

Keywords: Breast neoplasm; breast cancer; human epidermal growth factor receptor 2 (HER2); trastuzumab; meta-analysis

Submitted Nov 15, 2019. Accepted for publication Jan 10, 2020.

doi: $10.21037 /$ atm.2020.01.81

View this article at: http://dx.doi.org/10.21037/atm.2020.01.81 


\section{Introduction}

Human epidermal growth factor receptor 2 (HER2) is a member of the HER family and its overexpression and/ or gene amplification are known to be associated with aggressive tumour behaviour and poor breast cancer outcomes $(1,2)$. Trastuzumab is a humanized monoclonal antibody against HER2 and was the first anti-HER2 therapy developed (3-5). Anti-HER2 therapy, represented by trastuzumab, has been shown to improve both the diseasefree survival (DFS) and overall survival (OS) of patients with HER2-positive breast cancer in several randomized controlled trials (RCTs) (3-7).

Although the benefits of using trastuzumab have been demonstrated in patients who are node-positive or highrisk, node-negative $(3,5)$, its benefit is less clear for lowrisk, HER2-positive cases. Subgroup analyses of previous RCTs has provided information regarding the benefits of trastuzumab for treating early-stage breast cancers $(6,7)$; however, limited information is available regarding very lowrisk cases with tumours $<1 \mathrm{~cm}$ in size, without lymphatic metastases, and staged as T1abN0 according to the American Joint Committee on Cancer staging system (8).

With the recent universalization of screening, breast cancer is now commonly diagnosed at early stages (9). In clinical practice, it is not uncommon to encounter a situation where a decision is required on whether to administer trastuzumab for very low-risk breast cancer cases, such as TlabN0. The aim of this systematic review was to investigate the benefit of adjuvant treatment including trastuzumab in patients with HER2-positive breast cancer with tumours $<1 \mathrm{~cm}$ and without lymph node metastases (pT1abN0).

\section{Methods}

\section{Study protocol}

Our study adhered to the Preferred Reporting Items for Systematic Reviews and Meta-Analysis guidelines. A systematic search of databases, including PubMed, MEDLINE, and EMBASE, was performed to identify literature published before Sep 30, 2019. We designed the following search-engine term to identify studies of T1a or $\mathrm{b}$ and $\mathrm{N} 0$ breast cancer patients treated with/without trastuzumab: "breast cancer" AND trastuzumab AND (small OR T1ab OR "1 cm") AND ("local control” OR recurrence). Language or period restrictions were not applied. Conference abstracts were included only if they fully met the inclusion criteria. Ethical approval is not applicable because present study is a systematic review based on meta-analysis, which used only published data but not any data from human or other living organisms.

\section{Selection criteria}

The following criteria were used to identify suitable studies: (I) controlled clinical trials, retrospective studies, or prospective studies; and (II) at least five patients with T1a or b and N0 breast cancer treated with trastuzumab, with primary endpoint information. Our primary endpoint was tumour recurrence, local, distant, or any other recurrence type, as percentile rates or clinical descriptions. Clinical descriptions needed to be detailed enough to yield numerical percentiles. Studies using national databases or cancer registries, such as Surveillance, Epidemiology, and End Results, were not included to avoid duplicating patients and contradicting independent assumptions $(10,11)$.

The titles and citations of the reviews, editorials, in vivo and in vitro studies, case reports, and duplicated studies from the databases were initially screened by one researcher. Next, two independent researchers filtered the studies, removing those with irrelevant subjects or formats via abstract review. Full text reviews were performed by two independent researchers to identify studies that fully met the inclusion criteria. For studies published from a single institution, the study involving the largest number of patients with $\mathrm{T} 1 \mathrm{a}$ or $\mathrm{b}$ and $\mathrm{N} 0$ breast cancer treated with adjuvant treatment including trastuzumab and/or most recently published was included. After selection, the final set of studies included in the analysis was confirmed by the mutual consent of the two researchers.

\section{Data collection}

The following data were collected: (I) general information regarding the authors, year of publication, publication type, nationality, and study design and (II) clinical information regarding the number of target patients, chemotherapies used, and tumour recurrence. We did not collect survival data because the rates of survival were too high to statistically compare the effect of trastuzumab. The data collection process was performed by two independent researchers and any differences were resolved through mutual discussion. 
Table 1 General information of included studies

\begin{tabular}{|c|c|c|c|c|c|c|c|c|}
\hline Author & Year & Source & Publication type & Nationality & Affiliation & Study design & $\begin{array}{l}\text { Quality of } \\
\text { study by } \\
\text { NOS }^{a}\end{array}$ & $\begin{array}{l}\text { No. of } \\
\text { T1ab }\end{array}$ \\
\hline Tognela & 2015 & Clin Breast Cancer & Full article & Australia & 4 centers & Retrospective & High & 46 \\
\hline Olszewski & 2013 & $\begin{array}{c}\text { Breast Cancer Res } \\
\text { Treat }\end{array}$ & Full article & USA & $\begin{array}{c}\text { Brown University, Beth } \\
\text { Israel MC }\end{array}$ & Retrospective & High & 205 \\
\hline Fehrenbacher & 2014 & J Clin Oncol & Full article & USA & KPNC database & Retrospective & High & 234 \\
\hline Mcarthur & 2011 & Cancer & Full article & USA & MSKCC & Retrospective & High & 99 \\
\hline Gori & 2015 & PLos One & Full article & Italy & 25 centers & Retrospective & High & 303 \\
\hline Horio & 2012 & Int J Clin Oncol & Full article & Japan & Aichi Cancer Center & Retrospective & High & 42 \\
\hline
\end{tabular}

a, studies with a score $\geq 7$ were regarded as high quality and those with a score of 4-6, as medium quality. NOS, Newcastle-Ottawa scale; KPNC, Kaiser Permanente Clinical Care Program of North California; MSKCC, Memorial Sloan Kettering Cancer Center; MC, medical center.

\section{Quality assessment}

We used the Newcastle-Ottawa scale to assess study quality (1), as most studies were retrospective non-RCTs. Studies with a score of 7 or higher were regarded as high quality and those with scores between 4 and 6 were regarded as medium quality.

\section{Statistical analysis}

Pooled analyses of primary endpoints were performed using a random effects model (12), as the studies were designed and performed independently. Heterogeneity was assessed using a Cochran Q test (13) and I ${ }^{2}$ statistics (14). Significant heterogeneity was regarded to be present if the $\mathrm{P}$ value of the Cochran Q test was $<0.1$ and the $\mathrm{I}^{2}$ value was $>50 \%$. Publication biases were evaluated by visually inspecting the funnel plots and quantitative analyses using Egger's test (15). When the $\mathrm{P}$ value was $<0.1$, trimmed results using Duval and Tweedie's method were regarded as true outcomes. All statistical analyses were performed using Comprehensive Meta-Analysis software version 3 (Biostat, Inc., Englewood, NJ, USA).

\section{Results}

\section{Study inclusion and characteristics}

An initial search of different databases identified 720 studies. After exclusion based on the selection criteria, 280 studies were further processed by abstract review. After a review of the abstracts, 54 studies were further processed by fulltext review. Multiple studies published from one institution were filtered at this stage using the criteria listed in the Methods section. Finally, seven controlled studies that fully met the inclusion criteria were included in the present meta-analysis (16-22). These studies evaluated a total of 1,181 patients who were T1abN0, HER2-positive. Among them, 552 were treated with adjuvant treatment including trastuzumab and categorized as a trastuzumab group, and 629 were not treated including trastuzumab and categorized as a control group. All included studies were categorized as high quality and performed in community hospitals (studies were not performed with selected groups, such as nurses or volunteers), with included medical records (or multicentre hospital databases) and adequate follow-up to observe breast cancer recurrence (median follow-up periods of 37 78 months). Three of the 7 studies were conducted in the USA and one each was conducted in France, Australia, Italy, and Japan. General information regarding the included studies is summarized in Table 1.

\section{Recurrence rates and pooled analyses}

The median follow-up period ranged from 37 to 78 months. The information of adjuvant chemotherapy was available for 6 of 7 studies. Most patients in the trastuzumab group were also treated with concomitant chemotherapy. Most commonly used were anthracycline and taxane, and 75$100 \%$ of patients were treated with cytotoxic chemotherapy. In the control group, the rates of chemotherapy highly 
varied $(0-42 \%)$. Hormone therapy was administered in $20-63 \%$ and $24-66 \%$ of patients in the trastuzumab and control groups, respectively, whereas in the majority of the studies, $45-65 \%$ of patients were administered hormone therapy. Regarding clinicopathologic profiles, patients in the trastuzumab arm had generally inferior profiles such as higher rate of $\mathrm{T} 1 \mathrm{~b}$, grade 3 , and hormone negative cases, among available studies.

Of the 552 patients who underwent adjuvant treatment including trastuzumab among the seven available studies, ten cases of overall recurrence $(1.8 \%)$ were observed. Of the 629 control patients from the same studies, 55 $(8.7 \%)$ overall recurrences were reported. Among the five available studies with distant recurrence profiles, no distant recurrence was reported among 237 patients who underwent adjuvant treatment including trastuzumab. However, 16 cases of distant recurrence were reported among 436 control patients $(3.7 \%)$. These results are summarized in Table 2. Pooled analysis of overall recurrence for seven studies with 1,181 patients, showed that the odds ratio of trastuzumab treatment $v$ s. control was 0.201 [95\% confidence interval (CI): 0.100-0.404], with a $\mathrm{P}$ value $<0.001$, favoring the use of trastuzumab. No significant heterogeneity was identified among these studies for overall recurrence $\left(\mathrm{P}=0.479, \mathrm{I}^{2}=\sim 0.0 \%\right)$. For distant recurrence in five studies with 673 patients, the odds ratio of trastuzumab treatment vs. control was 0.328 (95\% CI: 0.082-1.311), with a $\mathrm{P}$ value of 0.115 . No significant heterogeneity was found in the analysis of distant recurrence $\left(\mathrm{P}=0.589, \mathrm{I}^{2}=\sim 0.0 \%\right)$. The results of pooled analyses are summarized in Table 3 and forest plots are presented in Figure 1.

\section{Publication bias}

No significant publication biases were found for overall or distant recurrence data. Egger's $\mathrm{P}$ values for pooled analyses of overall and distance recurrence were 0.175 and 0.495 , respectively. Funnel plots are shown in Figure 2.

\section{Discussion}

The benefits of anti-HER2 therapy using trastuzumab for patients with breast cancer have been demonstrated in several previous studies (3-5). Additionally, the benefits against cancer recurrence and for OS in node-positive or high-risk, node-negative patients have been shown in previous RCTs (5). Herceptin adjuvant (HERA) trial also showed a recurrence-free survival benefit, with a hazard ratio of 0.54 (95\% CI: $0.43-0.67, \mathrm{P}<0.0001$ ), although OS did not differ significantly (3). These pivotal trials concluded that chemotherapy with trastuzumab is essential in patients with large, node-positive, HER2-positive cancer $(3,5)$.

The benefits of trastuzumab against low-risk breast cancer remain unclear because of the favourable oncological outcomes of early-stage breast cancer and possible toxicities of trastuzumab (5). However, several studies have shown inferior oncological outcomes in HER2-positive cases compared to those in HER2-negative cases, suggesting that additional anti-HER2 treatment would be beneficial. Gonzalez-Angulo et al. analysed 965 patients with T1abN0 breast cancer and found that recurrence-free survival was significantly higher in HER2positive than in HER2-negative cases in the absence of adjuvant systemic therapy $(77.1 \%$ and $93.7 \%$, respectively, $\mathrm{P}<0.001)(23)$. According to a meta-analysis on pT1N0 patients by Zhou et al., HER2 positivity had a deleterious effect on DFS [risk ratio (RR) $=3.677,95 \%$ CI: $2.606-$ 5.189, $\mathrm{P}<0.001]$ and distant $\mathrm{DFS}(\mathrm{RR}=3.824,95 \% \mathrm{CI}$ : 2.249-6.501, $\mathrm{P}<0.001)$, compared to that in the hormone receptor $(\mathrm{HR})^{+} / \mathrm{HER}^{-}{ }^{-}$subgroup (24).

Subgroup analyses from previous RCTs have provided information regarding the benefits of trastuzumab use for treating early breast cancer. In the Breast Cancer International Research Group (BCIRG) 006 trial, the 5-year DFS rates were $72 \%, 86 \%$, and $86 \%$ for patients with pT1ab treated with doxorubicin and cyclophosphamide, followed by docetaxel (AC-T); AC-T plus trastuzumab; and docetaxel and carboplatin plus trastuzumab; respectively (AC-T plus trastuzumab: hazard ratio $=0.36, \mathrm{P}=0.03$; carboplatin plus trastuzumab: hazard ratio $=0.45, \mathrm{P}=0.09$ ). For node-negative cases, the 5-year DFS rates were $93 \%$ and $85 \%$ for the AC-T plus trastuzumab and AC-T groups, respectively (hazard ratio $=0.47, \mathrm{P}=0.003$ ) (6). In the HERA trial, the 3-year DFS rates among node-negative patients were $90.8 \%$ vs. $84.9 \%$ for the trastuzumab $v$. control groups (hazard ratio $=0.59$; 95\% CI: 0.39-0.91) (7). Although these results suggest a broad benefit of trastuzumab administration for low-risk breast cancer treatment, it is unclear whether trastuzumab treatment is beneficial for treating the very low-risk group of pT1abN0 cases.

Owing to the universalization of screening, early diagnosis of breast cancer is increasing (9). Therefore, it is not uncommon in clinical practice to encounter a situation where a decision is required regarding the use of trastuzumab to treat early-stage breast cancer cases, such 


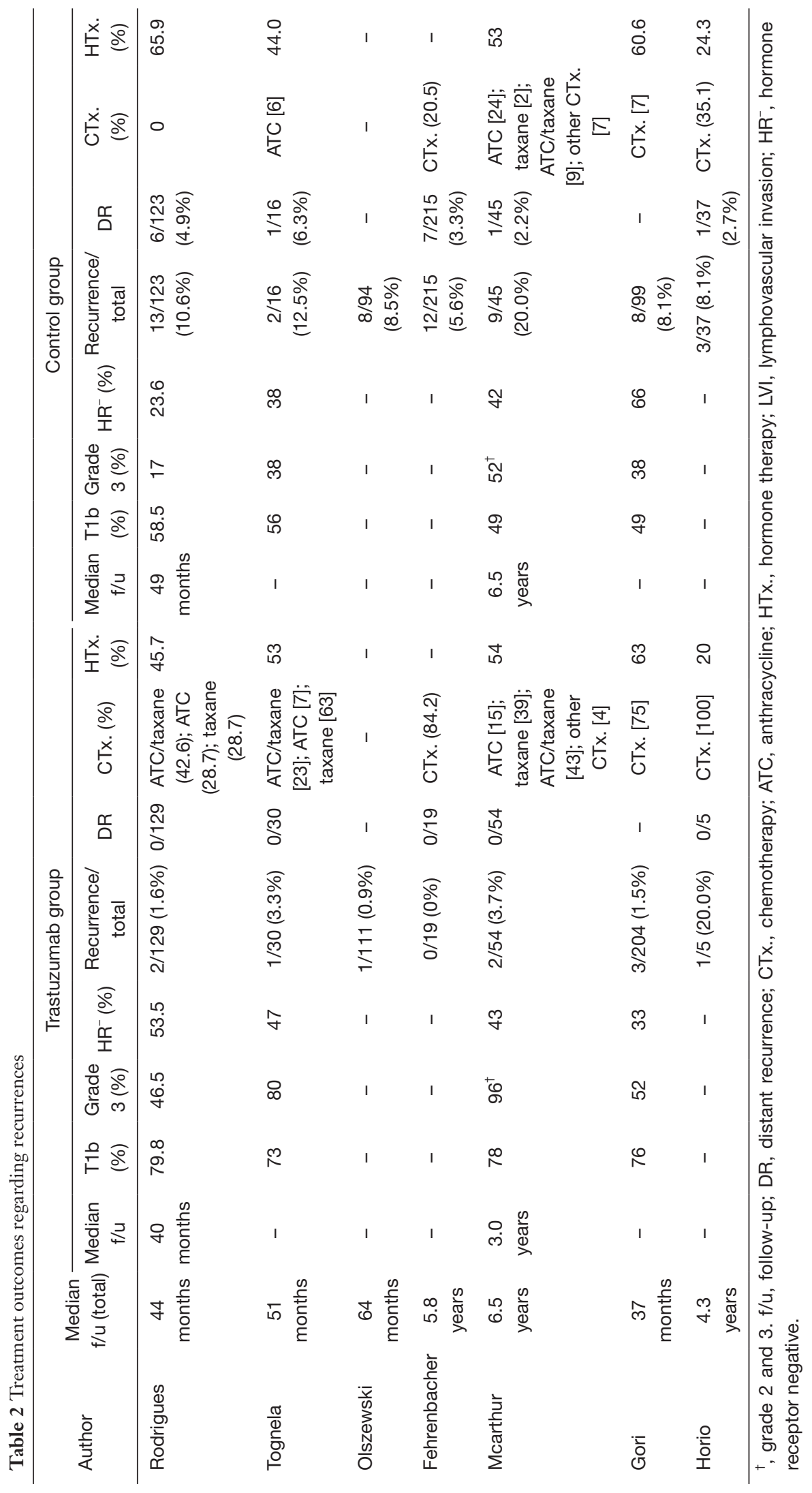


Table 3 Meta-analysis of recurrence

\begin{tabular}{|c|c|c|c|c|c|c|c|}
\hline $\begin{array}{l}\text { Recurrence } \\
\text { type }\end{array}$ & $\begin{array}{l}\text { No. of } \\
\text { studies }\end{array}$ & Total (n) & $\begin{array}{l}\text { Trastuzumab, } \\
\text { recur/total }\end{array}$ & $\begin{array}{l}\text { Control, } \\
\text { recur/total }\end{array}$ & $\begin{array}{l}\text { Trastuzumab vs. control, } \\
\text { odds ratio }(95 \% \mathrm{Cl})\end{array}$ & $P$ heterogeneity, $I^{2}$ & $P$ value \\
\hline $\begin{array}{l}\text { Overall } \\
\text { recurrence }\end{array}$ & 7 & 1,181 & $10 / 552(1.8 \%)$ & $55 / 629(8.7 \%)$ & $0.201(0.100-0.404)$ & $0.479,0.0 \%$ & $<0.001$ \\
\hline $\begin{array}{l}\text { Distant } \\
\text { recurrence }\end{array}$ & 5 & 673 & $0 / 237$ & $16 / 436(3.7 \%)$ & $0.328(0.082-1.311)$ & $0.589,0.0 \%$ & 0.115 \\
\hline
\end{tabular}

$\mathrm{Cl}$, confidence interval.

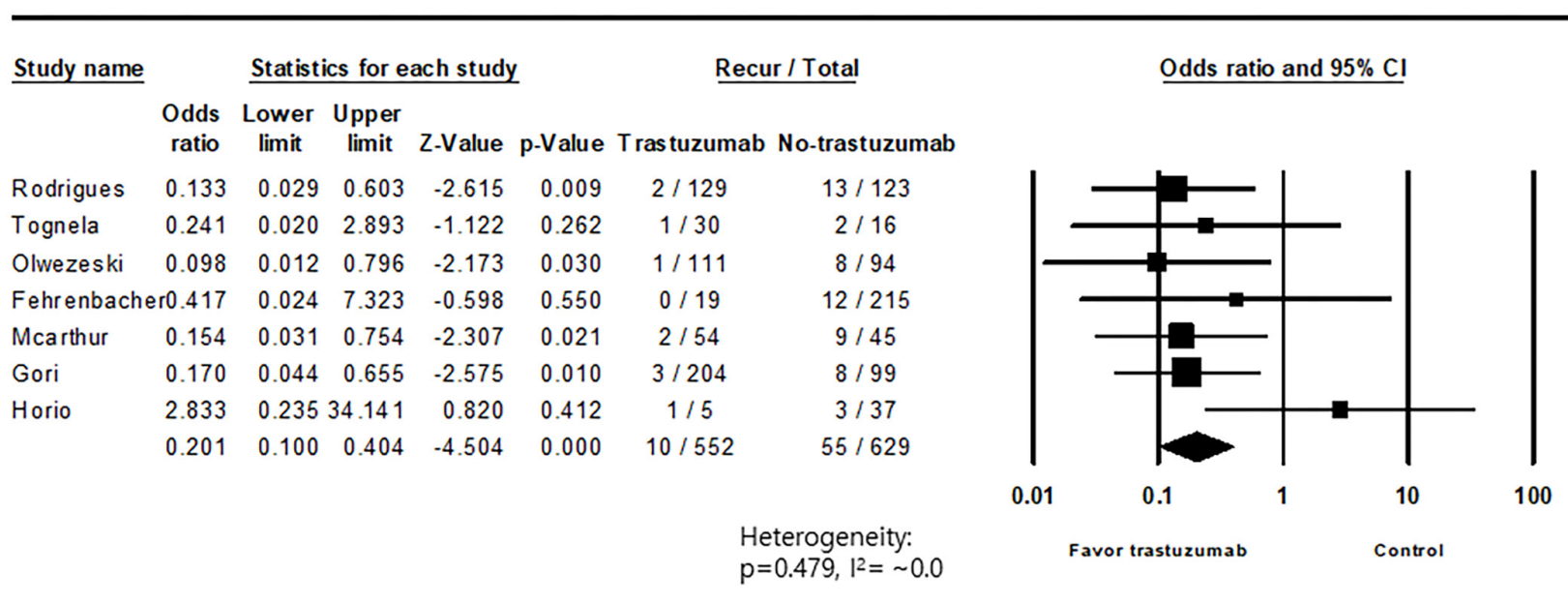

Distant recurrence

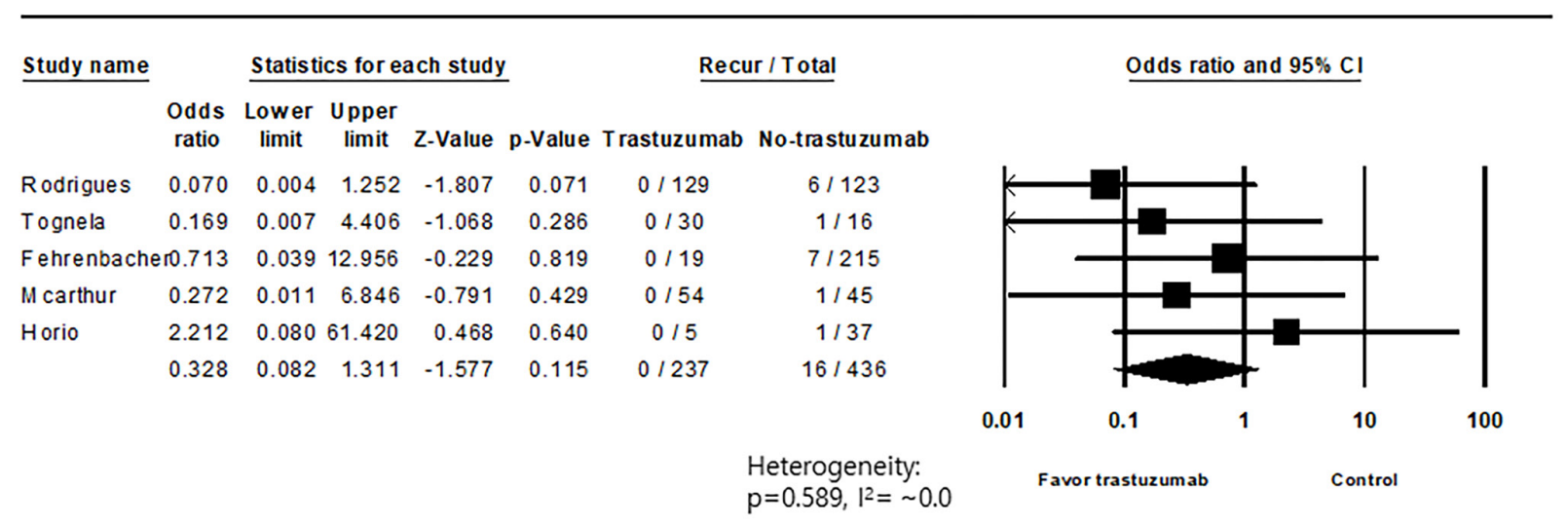

Figure 1 Forest plots of pooled analyses regarding overall and distant recurrences.

as pT1abN0. Recent National Comprehensive Cancer Network (NCCN) guidelines firmly recommend the use of adjuvant chemotherapy and trastuzumab for tumours $>1 \mathrm{~cm}$ or for node-positive, HER2-positive cases (category 1). The recommendation level is $2 \mathrm{~A}$ for $\mathrm{T} 1 \mathrm{bN} 0$ and $\mathrm{N} 1 \mathrm{mi}$ and $2 \mathrm{~B}$ for T1aN0. At these levels, the recommendation is to "consider adjuvant chemotherapy with trastuzumab". It is also stated that the decision to administer trastuzumab for $\mathrm{T} 1 \mathrm{ab}$ cases must be taken after considering other factors including possible cardiotoxicities and uncertain 

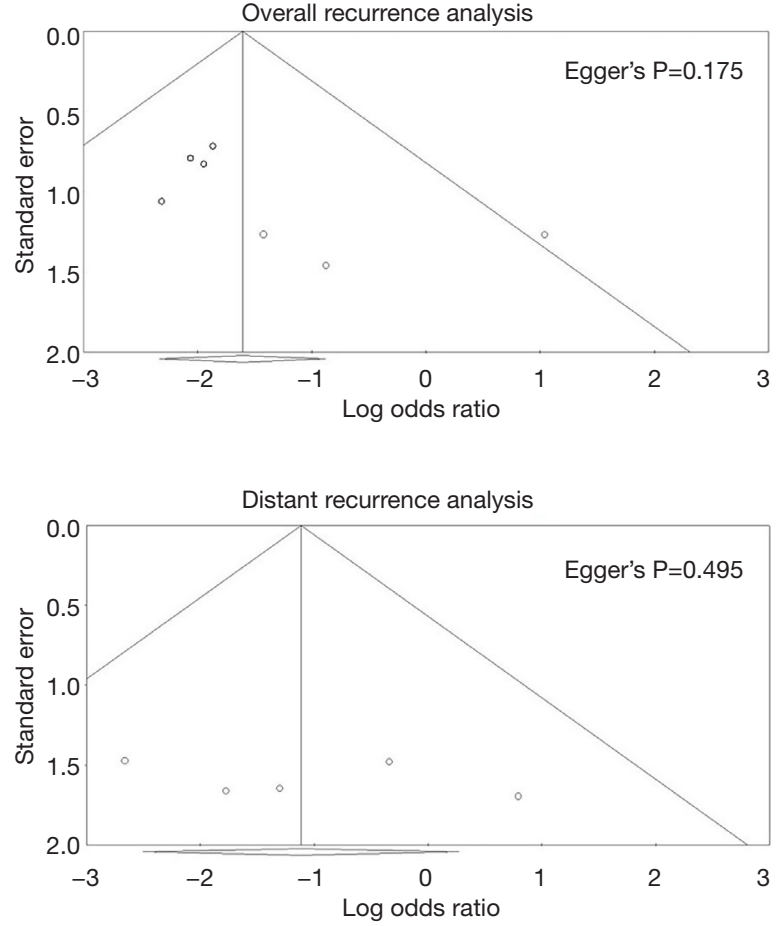

Figure 2 Funnel plots of pooled analyses regarding overall and distant recurrences.

benefits (25). In a recent Cochrane review, the RR was 5.11 (95\% CI: 3.00-8.72, $\mathrm{P}<0.00001$ ) for congestive heart failure and 1.83 (95\% CI: 1.36-2.47, $\mathrm{P}=0.0008$ ) for left ventricular ejection fraction decline (26).

Our meta-analysis provides clinically useful information on the benefits of trastuzumab for overall recurrence and possibly, distant recurrence. Although the difference in distant recurrence between trastuzumab-treated patients and controls was not statistically significant, it should be noted that no distant recurrence cases were reported among those who underwent trastuzumab treatment. It is a limitation of our study that we could not make a robust conclusion on the use of adjuvant treatment including trastuzumab, as we were only able to analyze the recurrence profile and not survival or cardiotoxicity. We expect that future controlled studies that take into consideration survival and toxicity, as well as recurrence, will yield more robust conclusions.

Zhou et al. (24) previously published a meta-analysis regarding adjuvant trastuzumab administration for pT1abN0 cases, reporting a benefit for DFS but not against locoregional recurrence. However, fewer than 500 patients from only four studies were pooled to analyse the efficacy of adjuvant trastuzumab, and two of the four studies were unpublished. The present study has the merit of including six published studies and one conference abstract, all with high quality design. Furthermore, our study involved $>1,100$ patients and included analyses of both overall recurrence and distant recurrence.

The most significant confounder that could not be controlled was concomitant chemotherapy; as the patients in the trastuzumab arm generally underwent more chemotherapy than those in the control, it is difficult to determine which treatment resulted in lower recurrence. However, in most studies, patients administered treatments including trastuzumab had inferior clinicopathologic profiles. In a study by Rodrigues et al. (16), which is one of the largest studies included, the trastuzumab arm had a higher rate of T1b (79.8\% vs. $58.5 \%)$, grade $3(46.5 \%$ vs. $17 \%)$, and lymphovascular invasion (22\% vs. $10.3 \%)$, higher mitotic index (23\% vs. $11.4 \%)$, and larger number of hormone negative $(53.5 \%$ vs. $23.6 \%)$ cases. Tognela et al. (17), Mcarthur et al. (20), and Gori et al. (27) found the same trends of higher rates of T1b, higher tumour grade, and more hormone-negative cases in their studies. Gori et al. conducted a large multicentre trial covering 25 Italian centres and found that most (66\%) patients were treated with systemic treatment including trastuzumab, reflecting the current clinical practice in Italy.

Another limitation of this study was that the studies evaluated were mostly observational. The metaanalysis of observational studies is controversial, as the heterogeneity among studies might affect pooled analyses and confounders cannot be fully controlled (28). However, a carefully conducted meta-analysis including assessments of study quality and publication bias might provide useful information in unrevealed area (28). Previous systematic reviews showed that meta-analyses of observational studies generally produce estimates of effect similar to those based on RCTs, and might improve the inference based on only RCTs (29). Although well-designed RCT provides the firmest evidence, the meta-analysis of observational studies might be the best possible way to provide clinically relevant information, for the subject with small effect size of endpoints where the RCT is difficult to be designed (30).

\section{Conclusions}

This study showed that adjuvant treatment including trastuzumab has significant overall recurrence benefits for pT1abN0, HER2-positive breast cancer patients. Although statistical significance was not reached, distant recurrence 
may be also reduced, as it did not occur among the 237 patients who underwent adjuvant treatment including trastuzumab. Differences in chemotherapy administration between the trastuzumab and control arms prevents definitive conclusions from being drawn, while inferior clinicopathologic profiles for patients in the trastuzumab arm suggesting a possible benefit of adjuvant treatment including trastuzumab. Although these results can aid in clinical decision-making by helping predict the benefits against recurrence, studies that also consider survival and toxicity are needed.

\section{Acknowledgments}

Funding: This work was supported by Korea University Ansan Hospital Grant (K1916671).

\section{Footnote}

Conflicts of Interest: The authors have no conflicts of interest to declare.

Ethical Statement: The authors are accountable for all aspects of the work in ensuring that questions related to the accuracy or integrity of any part of the work are appropriately investigated and resolved.

Open Access Statement: This is an Open Access article distributed in accordance with the Creative Commons Attribution-NonCommercial-NoDerivs 4.0 International License (CC BY-NC-ND 4.0), which permits the noncommercial replication and distribution of the article with the strict proviso that no changes or edits are made and the original work is properly cited (including links to both the formal publication through the relevant DOI and the license). See: https://creativecommons.org/licenses/by-nc-nd/4.0/.

\section{References}

1. Slamon DJ, Clark GM, Wong SG, et al. Human breast cancer: correlation of relapse and survival with amplification of the HER-2/neu oncogene. Science 1987;235:177-82.

2. Slamon DJ, Godolphin W, Jones LA, et al. Studies of the HER-2/neu proto-oncogene in human breast and ovarian cancer. Science 1989;244:707-12.

3. Piccart-Gebhart MJ, Procter M, Leyland-Jones B, et al. Trastuzumab after adjuvant chemotherapy in HER2- positive breast cancer. N Engl J Med 2005;353:1659-72.

4. Romond EH, Perez EA, Bryant J, et al. Trastuzumab plus adjuvant chemotherapy for operable HER2-positive breast cancer. N Engl J Med 2005;353:1673-84.

5. Perez EA, Romond EH, Suman VJ, et al. Four-year follow-up of trastuzumab plus adjuvant chemotherapy for operable human epidermal growth factor receptor 2-positive breast cancer: joint analysis of data from NCCTG N9831 and NSABP B-31. J Clin Oncol 2011;29:3366-73.

6. Slamon D, Eiermann W, Robert N, et al. Adjuvant trastuzumab in HER2-positive breast cancer. N Engl J Med 2011;365:1273-83.

7. Untch $M$, Gelber RD, Jackisch C, et al. Estimating the magnitude of trastuzumab effects within patient subgroups in the HERA trial. Ann Oncol 2008;19:1090-6.

8. Giuliano AE, Connolly JL, Edge SB, et al. Breast CancerMajor changes in the American Joint Committee on Cancer eighth edition cancer staging manual. CA Cancer J Clin 2017;67:290-303.

9. Smigal C, Jemal A, Ward E, et al. Trends in breast cancer by race and ethnicity: update 2006. CA Cancer J Clin 2006;56:168-83.

10. Borenstein M, Hedges LV, Higgins JP, et al. Introduction to meta-analysis. John Wiley \& Sons, 2011.

11. Shin IS. Same author and same data dependence in metaanalysis. Florida State University College of Education, 2009.

12. DerSimonian R, Kacker R. Random-effects model for meta-analysis of clinical trials: an update. Contemp Clin Trials 2007;28:105-14.

13. Cochran WG. The Combination of Estimates from Different Experiments. Biometrics 1954;10:101-29.

14. Higgins JP, Thompson SG. Quantifying heterogeneity in a meta-analysis. Stat Med 2002;21:1539-58.

15. Egger M, Davey Smith G, Schneider M, et al. Bias in meta-analysis detected by a simple, graphical test. BMJ 1997;315:629-34.

16. Rodrigues MJ, Peron J, Frenel JS, et al. Benefit of adjuvant trastuzumab-based chemotherapy in T1ab node-negative HER2-overexpressing breast carcinomas: a multicenter retrospective series. Ann Oncol 2013;24:916-24.

17. Tognela A, Beith J, Kiely B, et al. Small HER2-Positive Breast Cancer: Should Size Affect Adjuvant Treatment? Clin Breast Cancer 2015;15:277-84.

18. Olszewski AJ, Migdady Y, Boolbol SK, et al. Effects of adjuvant chemotherapy in HER2-positive or triplenegative pT1ab breast cancers: a multi-institutional 
retrospective study. Breast Cancer Res Treat 2013;138:215-23.

19. Fehrenbacher L, Capra AM, Quesenberry CP Jr, et al. Distant invasive breast cancer recurrence risk in human epidermal growth factor receptor 2-positive $\mathrm{T} 1 \mathrm{a}$ and $\mathrm{T} 1 \mathrm{~b}$ node-negative localized breast cancer diagnosed from 2000 to 2006: a cohort from an integrated health care delivery system. J Clin Oncol 2014;32:2151-8.

20. McArthur HL, Mahoney KM, Morris PG, et al. Adjuvant trastuzumab with chemotherapy is effective in women with small, node-negative, HER2-positive breast cancer. Cancer 2011;117:5461-8.

21. Gori S, Turazza M, Duranti S, et al. The Promher Study: An observational Italian study on HER2+ve, pT1a-b, pN0, M0 breast cancer (BC) patients (pts). Cancer Res 2015. doi: 10.1158/1538-7445.SABCS14-P5-18-05.

22. Horio A, Fujita T, Hayashi H, et al. High recurrence risk and use of adjuvant trastuzumab in patients with small, HER2-positive, node-negative breast cancers. Int J Clin Oncol 2012;17:131-6.

23. Gonzalez-Angulo AM, Litton JK, Broglio KR, et al. High Risk of Recurrence for Patients With Breast Cancer Who Have Human Epidermal Growth Factor Receptor 2-Positive, Node-Negative Tumors $1 \mathrm{~cm}$ or Smaller. J Clin Oncol 2009;27:5700-6.

Cite this article as: Lee HY, Shin IS, Rim CH. Benefits of adjuvant treatment including trastuzumab in HER2-positive pT1a-bN0M0 breast cancer: a systematic review and metaanalysis. Ann Transl Med 2020;8(5):187. doi: 10.21037/ atm.2020.01.81
24. Zhou Q, Yin W, Du Y, et al. For or against adjuvant trastuzumab for pT1a-bN0M0 breast cancer patients with HER2-positive tumors: a meta-analysis of published literatures. PLoS One 2014;9:e83646.

25. Mehta JL, Lopez LM. Rebound hypertension following abrupt cessation of clonidine and metoprolol. Treatment with labetalol. Arch Intern Med 1987;147:389-90.

26. Moja L, Tagliabue L, Balduzzi S, et al. Trastuzumab containing regimens for early breast cancer. Cochrane Database Syst Rev 2012;(4):CD006243.

27. Gori S, Inno A, Fiorio E, et al. The Promher study: an observational Italian study on adjuvant therapy for HER2-positive, pT1a-b pN0 breast cancer. PloS one 2015;10:e0136731.

28. Stroup DF, Berlin JA, Morton SC, et al. Meta-analysis of observational studies in epidemiology: a proposal for reporting. Meta-analysis Of Observational Studies in Epidemiology (MOOSE) group. JAMA 2000;283:2008-12.

29. Shrier I, Boivin JF, Steele RJ, et al. Should meta-analyses of interventions include observational studies in addition to randomized controlled trials? A critical examination of underlying principles. Am J Epidemiol 2007;166:1203-9.

30. Frieden TR. Evidence for Health Decision Making Beyond Randomized, Controlled Trials. N Engl J Med 2017;377:465-75. 